\title{
Orientierende Untersuchungen zur Anwendung der bildgebenden Ultraschallprüfung zur Fehlererkennung in Holz
}

\section{J. Neuenschwander}

Eidgenössische Materialprüfungs- und Forschungsanstalt (EMPA), 8600 Dübendorf/Schweiz

\section{P. Niemz, L. J. Kucera \\ ETH, 8092 Zürich/Schweiz}

Subject Studies for visualizing wood defects using ultrasonic in reflection and transmission mode

Material und Methode Die Ultraschalltechnik wird heute bereits vielfach in der Holzforschung zur Qualitätsbeurteilung genutzt. Dabei wird im wesentlichen die Durchschallung angewandt. Als Meßgröße wird fast ausschließlich die Laufzeit der Schallwellen genutzt. Da Defekte nur exakt erfaßt werden können, wenn diese in der Ausbreitungsrichtung der Schallwelle liegen, bereitet die zuverlässige Ortung von Defekten in größeren Bauteilen wie Brettern, Platten Probleme. Für die Detektierung von Fehlern in Metallverbindungen, Keramik etc. wird daher oft die bildgebende Ultraschallprüfung verwendet.

Versuchsmaterial Es wurden Messungen an Proben aus Vollholz (mit Ästen) und an einer Mitteldichten Faserplatte (MDF) mit in die Schmalflächen eingebrachten Bohrungen verschiedener Durchmesser durchgeführt. Die Schmalflächen wurden mit Klebeband wasserdicht versiegelt.

Versuchsmethodik Die Ultraschalluntersuchungen wurden in Tauchtechnik mit Wasser durchgeführt. Es wurden fokussierende Breitbandprüfköpfe mit Nominalfrequenzen von $0.5 \mathrm{MHz}$ und $2.25 \mathrm{MHz}$ verwendet. Gearbeitet wurde einerseits mit einem Prüfkopf mit der Impuls-Echo-Methode (Messung des Oberflächenechos der Probe) oder es wurde die Transmission mit getrenntem Sender und Empfänger gemessen (Durchschallung). Durch mäanderförmiges Abtasten (Scannen) der Probe wird ein Ultraschall C-Bild erzeugt.

Resultate Bild 1 zeigt das nach der Impuls-Echo-Methode bei 2.25 MHz ermittelte Abbild der Holzoberfläche. Äste und auch die Jahrringstruktur können sowohl beim Scannen der Breit(Bild 1a) als auch der Schmalfläche recht gut erkannt werden (Bild 1b). Der Grund für die Ortsabhängigkeit der Echoamplitude liegt in der unterschiedlichen akustischen Impedanz von Früh-, Spät- und Astholz.

Innenliegende Defekte wie Bohrungen sind schwierig nachzuweisen, da im Holz die Schallschwächung sehr hoch ist. Dies zeigte sich sowohl bei Naturholz als auch bei MDF. Bild 2 zeigt ein Durchschallungs C-Bild bei $0.5 \mathrm{MHz}$ der $18 \mathrm{~mm}$ dicken MDFProbe. Man erkennt zwar eine gewisse Korrelation aber sicher nicht ein getreues Abbild der eingezeichneten Bohrungen.

Prinzipiell bietet die bildgebende Ultraschallprïfung eine Reihe von Vorteilen im Vergleich zur üblichen „linienförmigen“ Mes-

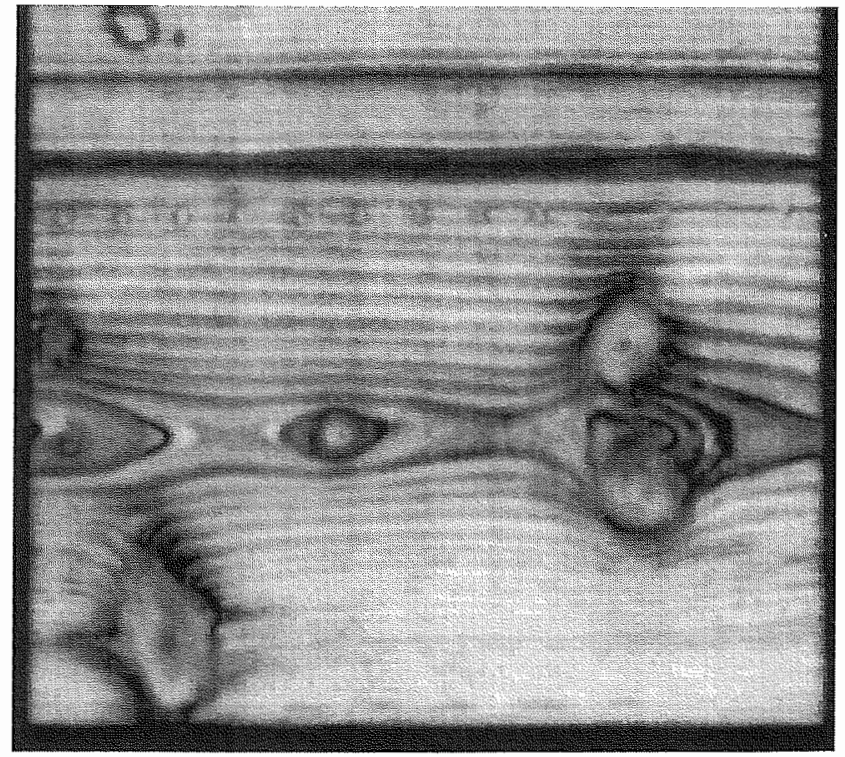

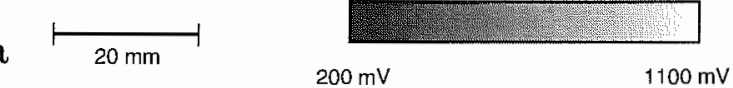

$200 \mathrm{mV}$

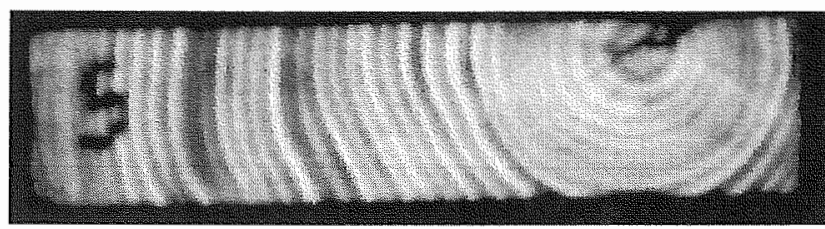

b
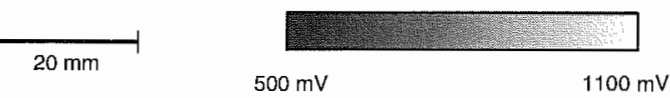

Bild 1. Durch Ultraschall-Messung ermitteltes Abbild der Holzoberfläche (Kiefer mit Ästen) Meßgröße: Reflexion der Oberfläche; a Breitfläche; b Schmalfäche

Fig. 1. Ultrasonic imaging of a wood surface in reflection mode (pine containing knots): a face side (radial view); b narrow surface (cross section)

sung, z.B. die präzise Ortung und Analyse von Defekten und die Dokumentation des Befundes mit einem Bild.

$\mathrm{Zu}$ lösende Hauptprobleme sind

- die trockene Ankopplung

- die Messung im Durchschallungsmodus

- die Optimierung der Meßfrequenz 


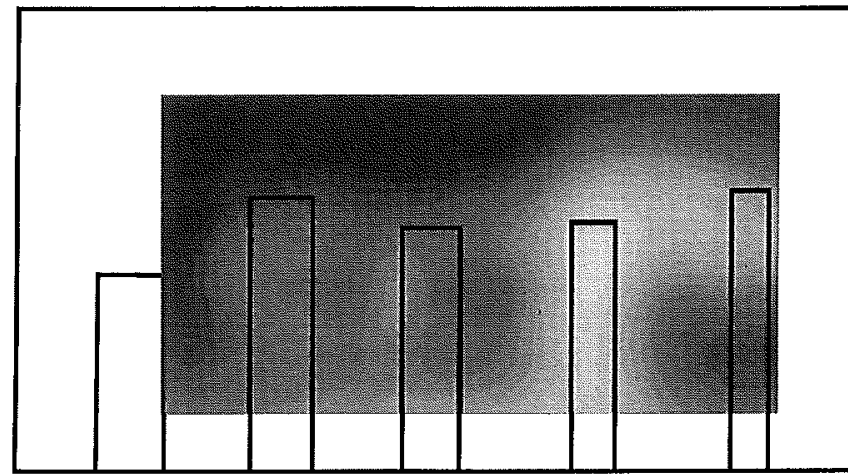

\section{Literatur}

Schob, M. et al. 1997: Untersuchungen zum Einfluß von Fehlergröße und Meßanordnung auf die Schallausbreitungsgeschwindigkeit in Holz. Holz Roh-Werkstoff 55: (im Druck)

Gilmore, R. S. 1996: Industrial ultrasonic imaging and microscopy. J. Phys. D: Appl. Phys. 29: 1389
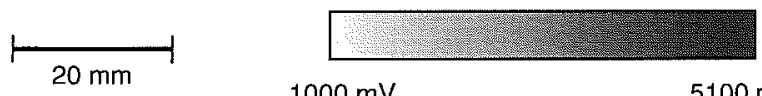

$1000 \mathrm{mV}$

$5100 \mathrm{mV}$

Bild 2. Durch Ultraschall-Messung ermitteltes Abbild einer MDFProbe mit innenliegenden Defekten (Bohrungen mit verschiedenen Durchmessern). Meßgröße: Transmittierter Impuls

Fig. 2. Ultrasonic image of an MDF sample containing bore holes of different diameters. Transmission mode 